\title{
THROMBOEMBOLIC DISEASE IN CHILDREN (EPIDEMIOLOGY, ETIOPATHOGENY AND DIAGNOSIS) (I)
}

\author{
Otilia-Elena Frasinariu, Violeta Streanga, Aniela Rugina, Irina Ciomaga, \\ Nistor Nicolai \\ Pediatrics Department, "Gr. T. Popa” University of Medicine and Pharmacy, Iasi
}

\begin{abstract}
Thrombosis is the result of an imbalance between two complex systems: hemostasis and fibrinolysis. Although
the incidence of thromboembolism is lower in children than in adults, the associated morbidity is clinically rele-
vant. This paper summarizes the main risk factors - important in defining effective strategies for primary throm-
boprophylaxis in children at risk, the diagnostic criteria and the optimal therapeutic approach, which have
mostly been extrapolated from the adult's recommendations until now.
\end{abstract}
Keywords: thrombosis, child, anticoagulant therapy

\section{INTRODUCTION}

Thromboembolic disease is generally considered an adult pathology; its incidence has been increasing among pediatric population for the past two decades $(1,2,3)$. Thrombosis in children are correlated with significant morbidity and mortality rates, including the recurrence of venous thromboembolism, the post-thrombotic syndrome, as well as death caused by massive pulmonary thromboembolism (4).

\section{EPIDEMIOLOGY}

Venous thromboembolism (VTE) includes deep venous thrombosis (DVT), from the upper and lower limb or from the central vasculature, pulmonary thromboembolism (PTE), cerebral venous sinus thrombosis (CVST) and renal vein thrombosis (RVT). VTE is rare in children compared to adult, but it is increasingly recognized as a complication of intensive care therapeutic measures for previously lethal childhood diseases (5). VTE incidence is not precisely known due to a limited number of studies. Data reported by Andrew M. et al. in Canada (6), van Ommen H. et al. in the Netherlands (7) and Stein PD in the USA (3) indicate an incidence ranging between $0.07-0.49$ per 10,000 children aged 1 month to 18 years, or 5.3-8.7 per 10,000 hospitalized children. However, a study from USA reported a 70 percent increase of VTE incidence between 2001 and 2007, from 34 cases per 10,000 children to 58 cases per 10,000 children in tertiary care hospitals (2). Currently, in Romania there are no studies that indicate the incidence and prevalence of this disease in children.

Two peaks of incidence have been reported: during the first year of life and during adolescence (3). The higher incidence in adolescents is mainly due to the transition to a coagulation profile similar to the one in adults. In adolescents, VTE is usually multi-factorial, the majority of cases presenting at least two risk factors (RF) at the time of diagnosis, suggesting the necessity of a detailed evaluation for risk factors in this population (4). In adolescents, the incidence is higher in girls than in boys ( $1.49 \mathrm{vs}$. $0.81 / 10,000$ children/year), pregnancy and the use of oral contraceptives enhancing this difference (3).

In children the majority of venous thrombosis (VT) are acquired. At least one risk factor is present in over $90 \%$ of children affected by thrombosis (4). Spontaneous or idiopathic thrombosis are rare. Data from the Canadian and Dutch registers showed that their frequency is only $2-8.5 \%$ of total pediatric venous thrombosis, unlike $40 \%$ in the adult population $(7,8)$. Recording the different types of VTE's, studies have shown a preponderance of DVT and PE, with an incidence of $90-96 \%$ of the total thromboembolic events (4). 
The mortality rate of children with thrombosis is between $6.4-17 \%$, but it is usually correlated with the associated pathology (9). A recent study mentions a general mortality of 11.4 per 1,000 children/ year (10), only $1.5-2.2 \%$ being directly correlated to VTE (7). Death risk decreases with increasing age, due to the fact that VTE in young children often appears during critical diseases (5).

The incidence of arterial thromboembolism (ATE) is 8.5 in 10,000 hospitalized children (11). In Denmark, the incidence of ischemic stroke was 1.33 per 100,000 persons/year, with a peak of incidence in children younger than 1 year (1). The majority of the arterial thrombosis in children are iatrogenic, secondary to an arterial catheter. Also, recent studies show an incidence of 3.4-11.4\% of arterial thrombosis after cardiac catheterization, young age and low weight being associated with an increased risk $(11,12)$.

\section{ETIOPATHOGENY OF THE THROMBOEMBOLIC DISEASE}

The hemostatic and fibrinolytic systems in children are continuously evolving through adolescence, presenting certain peculiarities compared to adult's. Plasmatic levels of vitamin $\mathrm{K}$ dependent coagulation factors (II, V, VII, IX, X, XI, XII) are $20 \%$ lower than in adults (3). Also, up to the age of 6 , plasma levels of plasminogen, $\alpha 2$ anti-plasmin and tissue plasminogen activator (tPA) are reduced compared to those in adults. The plasminogen activator inhibitor (PAI-1) levels are increased. During childhood, antithrombin III and protein C levels are significantly lower compared to those in adults, while protein $S$ values reach similar adult levels by the age of 3-6 months (13).

Several factors may protect children from TE, thereby contributing to its low incidence during childhood. Among these factors, the most important are: reduced thrombin generation capacity, increased capacity of the alpha-2-macroglobulin to inhibit thrombin and the presence of circulating anticoagulants at birth. There is an increased endothelial antithrombotic potential; the diseases evolving with endothelial lesions (diabetes, dyslipidemia, hypertension) are rare in children. Moreover, children are less frequently exposed to acquired prothrombotic risk factors (malignancy, oral contraceptives, hormone replacement therapy, pregnancy, smoking, surgery) (14).

The primary mechanism for venous thrombosis is considered the Virchow's triad: venous stasis, en- dothelial lesion and hypercoagulability state; the contribution of each factor varies according to the age group. The hypercoagulant state may be due to a primary disease, thrombophilia, or may be secondary to other diseases or therapies (contraceptives, hormone therapy, and chemotherapy) (15).

The etiology of thrombosis in children varies and there are a lot of risk factors (Table 1). Thrombosis is often precipitated by the coexistence of several risk factors.

TABLE 1. The main risk factors of thrombosis in children

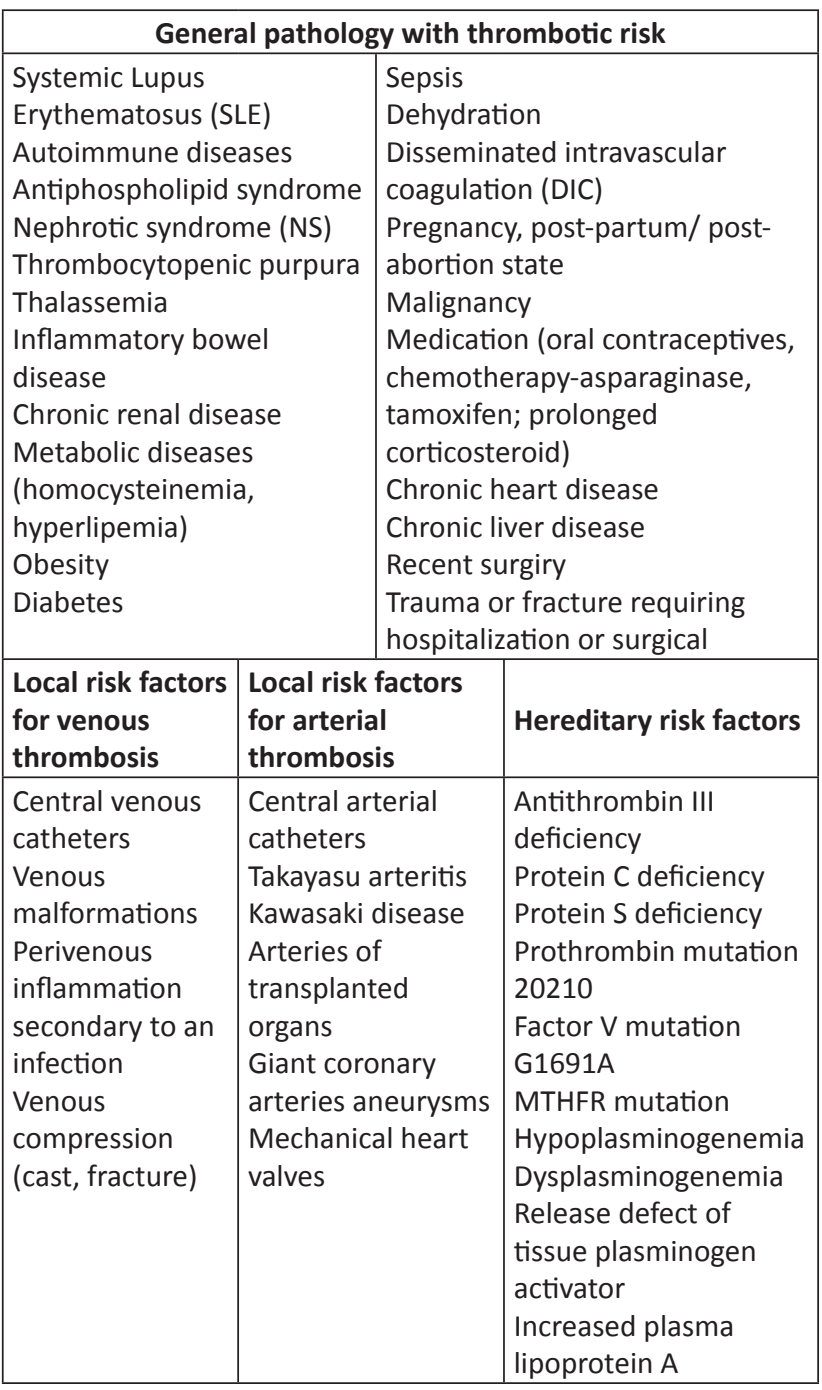

CVC is considered the most common cause associated with TE. Thrombosis correlated with a catheter represents $90 \%$ of all thrombotic events during the first year of life and up to two thirds of the total VTE's in children $(6,7)$. The majority of the CVC-associated venous thrombosis occur in the upper venous system, due to the presence of a CVC through a jugular or subclavian vein. Most cases of arterial thrombosis have an underlying pathology. Arterial thromboembolic events that are unrelated to catheters are due to a congenital condi- 
tion (hyperhomocysteinemia, primary hyperlipidemia, cardiopathy) or to an acquired one (Table 1).

Hereditary thrombophilia occurs in $10-59 \%$ of children with idiopathic venous thrombosis $(1,16,17)$. The most significant hereditary diseases with prothrombotic effect are illustrated in table I. Studies in children with VTE have shown an incidence of the primary hereditary anomalies of: 4.7$13 \%$ for the presence of factor V Leiden; $2.3-3 \%$ for prothrombin gene mutation; $1-1.2 \%$ for protein $\mathrm{S}$ deficiency; $1 \%$ for antithrombin III deficiency; $0.6-1 \%$ for protein $\mathrm{C}$ deficiency; $2.6 \%$ for combined defects and $7.5 \%$ for the increased plasma lipoprotein A concentration (16,17). A meta-analysis of 22 observational studies showed a significant association of thrombophilia with the occurrence of ischemic stroke and cerebral venous sinus thrombosis, with a summary odds ratio ranging from 2.43 for the prothrombin gene mutation to 9.31 for protein $\mathrm{C}$ deficiency and 11.86 in the presence of at least 2 risk factors (18).

Thrombophilia is an additional risk factor, not a unique trigger factor in the thrombotic pathology. The thrombotic risk in a healthy child who has just one thrombophillic defect is low, acquired risk factors playing a major contribution. Routine screening for thrombophilia in healthy children under the age of 15 , members of families with inherited thrombotic disorders, is not justified, as the real risk of VTE is low (19). The presence of thrombophilia is correlated with an early age of TE onset and with frequent recurrences. Recurrent VTE is associated with all the hereditary clotting disorders, except for the increased levels of A lipoprotein (20).

Activated protein $C$ resistance (APCR), as a result of the mutation of the factor V Leiden, is considered the most frequent hereditary predisposition to thrombosis, and is found in $3-8 \%$ of the population (15). The disease is autosomal dominant (AD) transmitted. The risk of thrombosis is 7-8-fold increased in heterozygous cases compared to the general population; the risk increases up to 80 times in homozygous cases. It mainly manifests through VT, recurrent thromboembolism, including cerebral venous thrombosis, and repeated spontaneous abortions in adults (15). APCR is often associated with other genetic predispositions (deficiency of protein $\mathrm{C}$, protein $\mathrm{S}$, antithrombin III).

Proteins $\mathrm{C}$ and $\mathrm{S}$ are vitamin-K dependent proteins which inactivate factors Va and VIIIa. The incidence in the general population is $0.14-0.5 \%$ (15). Protein C or S deficiency is AD transmitted. The most common manifestations of protein $\mathrm{C}$ deficiency are VT of the inferior limbs, iliofemoral veins, mesenteric veins, cerebral venous thrombosis, purpura fulminans in homozygous newborns, and cutaneous necrosis induced by treatment with warfarin in heterozygous patients. Arterial thrombosis is more frequent during adulthood. Protein $\mathrm{S}$ deficiency is manifested by DVT, superficial thrombophlebitis and PTE.

Antithrombin III deficiency is AD transmitted, with a $0.02-0.17 \%$ incidence in the general population (15). Homozygous forms manifest dramatically, with extended thrombosis from the first hours after birth, leading to death. The risk of thrombosis is estimated at $50 \%$ (16). In the case of liver diseases, NS, CID, and treatment with oral contraceptives, an acquired antithrombin deficiency may occur (15). AT III deficiency is most commonly associated with VT.

Antiphospholipid antibodies (cardiolipin antibodies, lupus anticoagulant) are associated with hypercoagulant syndrome. TE incidence in SLE patients is between $9.2-17 \%$ (7).

The main complications of thromboembolism are recurrent thrombosis, post-thrombotic syndrome, bleeding and death. Increased plasmatic levels of factor VIII and/or D-dimers and the persistence of these values after standard anticoagulation therapy indicate a poor prognosis in children with thrombosis (21). The recurrence rate is $4.8 \%$ in children with no genetic risk factors, $17.6 \%$ in the presence of one hereditary risk factor, rising up to $50 \%$ in the presence of more than two hereditary RF (20). Post-thrombotic syndrome is characterized by chronic venous insufficiency, symptoms varying from edema to chronic pain and ulceration of the affected limb. Data from the Canadian registry reveal a $21-25 \%$ prevalence in children with VT (22).

\section{DIAGNOSIS}

The diagnosis of thrombosis is mainly clinical, the investigations should be completed by biological and paraclinical tests, depending on the suspected etiology.

A well conducted history may reveal a possible thrombotic cause, by assessing thrombotic risk factors. Clinical manifestations are varied and depend on the localization and extension of the thrombus. In children, signs and symptoms may be similar or overlap with those occurring in other pathologies, more frequently encountered and easier to suspect at the first check-up. Also, symptoms may be absent or so discrete that they cannot be highlighted in clinical examination. The incidence of asymp- 
tomatic VTE associated with a $\mathrm{CVC}$ is estimated to be $66 \%$ of all children with CVC and up to $40 \%$ of pediatric oncologic patients (22).

In deep vein thrombosis a painful edema and erythema of the limb can be noticed, usually in the lower extremities. Positive Homans sign and a painful cord on palpation can be noticed at the lower limb (pain on dorsiflexion of the foot on the leg). On the upper limb, the thrombosis extension to the superior vena cava (VCS) leads to superior vena cava syndrome with cape edema, chemosis, facial edema, collateral venous circulation and cyanosis in the VCS territory. Furthermore, chylothorax or chylopericardium may also occur (23). Symptomatic CVC-related VTE can be manifested through repeated loss of CVC's permeability, sepsis and collateral cutaneous circulation. Arterial thrombosis of the limbs may cause diminished peripheral pulse, low local temperature and progressive change in color from purple to black.

Pulmonary thromboembolism can be suspected if violent thoracic pain (often retrosternal or precordial), marked dyspnea, cyanosis, tachycardia or syncope occurs. Persistent tachypnea without an obvious cause is an important symptom appearing in all ages (22).

Cerebral arterial and venous thrombosis can by asymptomatic in the newborn or may manifest with headache, vomiting, seizures, focal neurologic deficits, sensory loss, and hemiparesis in children.

In the case of renal vein thrombosis, the clinical examination may reveal an abdominal mass on deep palpation, macroscopic hematuria with or without oligo-anuria, proteinuria or arterial hypertension. Risk factors associated with RVT are NS, SLE, and renal transplantation. Rarely, if the thrombus is located in the splanchnic territory (portal, splenic or mesenteric veins), thrombosis is manifested acutely with abdominal pain, signs of liver failure, gastrointestinal bleeding or it may be asymptomatic. The underlying contributing factors are local malignancies, cirrhosis, splenectomy, infections, and presence of antiphospholipidic antibodies (24).

In severe cases, such as chronic myeloproliferative disease, DIC, anti-K vitamin induced necrosis, an extended arteriovenous thrombosis, with severe symptoms up to large cutaneous necrosis or limb necrosis, may occur.

Hereditary thrombophilia is suspected in case of recurrent thrombosis without associated risk factors, with a positive family history of thrombosis.

There are several methods of confirming thrombosis and assessing its extension and severity.
There are no specific laboratory tests available for TE diagnosis. Anemia, thrombocytopenia or hematic fragments may suggest DIC. Recommended investigations for assessing the coagulation status are: coagulation time, prothrombin time, prothrombin activity, partial thromboplastin time (PTT), partial activated thromboplastin time (APTT), INR and fibrinogen. Acute thrombosis is accompanied by increased plasmatic levels of some coagulation factors (fibrinogen, V, VII, VIII, vWF, X). The presence of antiphospholipidic antibodies (anticardiolipin antibodies, anti- $\beta 2$-glycoprotein 1 antibodies) and lupus anticoagulant may also be investigated.

To establish the thrombophilic etiology, some abnormalities should be looked for: factor V Leiden mutation, prothrombin gene mutation 20210A, antithrombin III deficiency, protein $\mathrm{C}$ and protein $\mathrm{S}$ deficiency, homocysteinemia and increased lipoprotein A levels. In the absence of those abnormalities, if the suspicion of hereditary hemostasis anomalies persists, the factors IX, XI and a dysfibrinogenemia will be investigated. Plasma levels of anticoagulant factors (antithrombin III, proteins C, protein S) may be affected by the acute thrombotic process or by the anticoagulant therapy (25).

Increased serum level of D-dimer in the presence of an acute thrombosis is due to simultaneous activation of coagulation and fibrinolysis. D-dimers dosage is a very sensitive diagnosis tool, but less specific. D-dimers can also rise during inflammatory, infectious, tumoral and traumatic pathologys, post-surgery, during pregnancy or liver disease. A normal level excludes the possibility of a DVT or an acute PTE. The dosage of D-dimers is very useful for surveying thromboembolic disease, in order to assess recurrences (26).

Diagnostic imaging methods for establishing the location and extension of the thrombotic process are based on invasive (venography) or noninvasive (Doppler-US, CT scan) techniques. Doppler ultrasound is used as a primary diagnostic tool to confirm thrombosis in adults and children, the results being comparable with those offered by contrast angiography. The Doppler signal is absent in the thrombosed vessels, and the lumen cannot be compressed. Ultrasound can distinguish between a recent and an old thrombosis (25). Factors that could interfere with this imaging method in the pediatric population include: the small diameter of the vessels, decreased pulse pressure, and the presence of a venous line where the thrombus is located, which makes it difficult to compress the vein (15). Doppler ultrasound is recommended for the 
initial assessment of the lower extremities thrombosis. Except for jugular and axillary veins, Doppler ultrasound has a low sensibility when applied for diagnosis of thrombosis in the upper venous system, leading to false-negative results (23).

Contrast angiography is considered the gold standard for the diagnosis of thrombosis in children. Today it is rarely used, because it is an invasive, painful technique and the peripheral access is difficult to obtain in children (25). In case of a highly clinical suspicion of thrombosis associated with a negative Doppler ultrasound evaluation, a $\mathrm{CT}$ angiography or MRI investigation can be performed to confirm the thrombosis. Craniocerebral contrast CT scan is useful for detecting a cerebral

\section{REFERENCES}

1. Tuckuviene R., Christensen A.L., Helgestad J. et al. Paediatric arterial ischaemic stroke and cerebral sinovenous thrombosis in Denmark 1994-2006: a nationwide population-based study. Acta Paediatr. 2011; 100(4):543-9.

2. Raffini L., Huang Y.S., Witmer C., Feudtner C. Dramatic increase in venous thromboembolism in children's hospitals in the United States from 2001 to 2007. Pediatrics. 2009; 124(4):1001-8.

3. Stein P.D., Kayali F., Olson R.E. Incidence of venous thromboembolism in infants and children: data from the National Hospital Discharge Survey. J Pediatr 2004; 145:563-5.

4. Goldenberg N.A., Bernard T.J. Venous thromboembolism in children. Hematol Oncol Clin North Am 2010; 24:151-66.

5. Chalmers E.A. Epidemiology of venous thromboembolism in neonates and children. Thromb Res 2006;118:3-12.

6. Andrew M., David M., Adams M., et al. Venous thromboembolic complications (VTE) in children: first analyses of the Canadian Registry of VTE. Blood 1994; 83:1251.

7. van Ommen C.H., Heijboer H., Büller H.R., et al. Venous thromboembolism in childhood: a prospective two-year registry in The Netherlands. J Pediatr 2001; 139:676.

8. Schmidt B., Andrew M. Neonatal thrombosis. Report a prospective Canadian and international registry. Pediatrics 1995; 96: 939-943.

9. Monagle P., Adams M., Mahoney M. et al. Outcome of pediatric thromboembolic disease: a report from the Canadian Childhood Thrombophilia Registry. Pediatr Res. 2000; 47:763-766.

10. Sabapathy C.A., Djouonang T.N., Kahn S.R., Platt R.W., Tagalakis V. Incidence Trends and Mortality from Childhood Venous Thromboembolism: A Population-Based Cohort Study. J Pediatr. 2016; 172:175-180.

11. Wang J.J., Shi K.L., Li J.W., Jiang L.Q., Kaspi O. et al. Risk factors for arterial ischemic and hemorrhagic stroke in childhood. Pediatr Neurol 2009; 40: 277-281.

12. Brotschi B., Hug M.I., Kretschmar O., Rizzi M., Albisetti M. Incidence and predictors of cardiac catheterisation-related arterial thrombosis in children. Heart. 2015; 101(12):948-53.

13. Andrew M., Vegh P., Johnston M., et al. Maturation of the hemostatic system during childhood. Blood 1992; 80:1998.

14. Spentzouris G., Scriven R.J., Lee T.K., Labropoulos N. Pediatric venous thromboembolism in relation to adults. J Vasc Surg. 2012; 55(6):1785-93. venous sinus thrombosis, but the MRI scan is more sensible in detecting an early arterial stroke (25).

Ventilation-perfusion scintigraphy is a standard diagnostic test used for suspected PTE, but it has been widely replaced by CT/MRI angiography in children (25). The correlation between elevated Ddimers levels and an altered appearance of the scanning imaging confirms PTE diagnosis. It is important to note that pulmonary scintigraphy is preferred in pregnant teenagers to CT angiography, because of a lower fetal radiation absorption (26). Electrocardiography, chest radiography and assessment of arterial blood gases can be used to evaluate suspected PTE, in order to exclude a different pathology, but cannot confirm or rule out the presence of thrombosis.

15. Anderson Jr F.A., Spencer F.A. Risk Factors for Venous Thromboembolism. Circulation. 2003; 107: 9-16.

16. van Ommen C.H., Heijboer H., van den Dool E.J., et al. Pediatric venous thromboembolic disease in one single center: congenital prothrombotic disorders and the clinical outcome. J Thromb Haemost 2003; $1: 2516$.

17. Revel-Vilk S., Chan A., Bauman M., Massicotte P. Prothrombotic conditions in an unselected cohort of children with venous thromboembolic disease. J Thromb Haemost 2003; 1:915.

18. Kenet G., Lütkhoff L.K., Albisetti M. et al. Impact of thrombophilia on risk of arterial ischemic stroke or cerebral sinovenous thrombosis in neonates and children: a systematic review and meta-analysis of observational studies. Circulation. 2010; 121(16):1838-47.

19. De Stefano V., Rossi E. Testing for inherited thrombophilia and consequences for antithrombotic prophylaxis in patients with venous thromboembolism and their relatives. A review of the Guidelines from Scientific Societies and Working Groups. Thromb Haemost. 2013; 110(4):697-705.

20. Nowak-Göttl U., Junker R., Kreuz W., et al. Risk of recurrent venous thrombosis in children with combined prothrombotic risk factors. Blood 2001; 97:858.

21. Goldenberg N.A., Knapp-Clevenger R., Manco-Johnson M.J. Elevated plasma factor VIII and D-dimer levels as predictors of poor outcomes of thrombosis in children. N Engl J Med. 2004; 351:1081-1088.

22. Albisetti M., Chan A. Venous thrombosis and thromboembolism in infants and children: Risk factors and clinical manifestations. http:// www.uptodate.com.

23. Gherasim L. Ghid de diagnostic şi tratament al tromboembolismului venos. Medicină internă 2008; 4 :

24. Michot C., Bloude R., Sachs P. Thromboses en pediatrie. In Dauger $S$, Leteurtre $S$, Beaufils $F$ (eds) Reanimation pediatrique. Doin editeurs 2010 225-238.

25. Chalmers E., Ganesen V., Liesner R., et al. Guideline on the investigation, management and prevention of venous thrombosis in children. Br J Haematol 2011; 154:196.

26. Konstantinides S., Torbicki A., Agnelli G. et al. ESC Guidelines on the diagnosis and management of acute pulmonary embolism. Eur Heart J 2014; 35: 3033-3080. 